\title{
Assessment and referral in suspected COVID-19: a community based observational study
}

Samuel Finnikin ( $\nabla$ s.j.finnikin@bham.ac.uk)

University of Birmingham

Shamil Haroon

University of Birmingham

Jennifer Cooper

University of Birmingham

Astha Anand

University of Birmingham

Abijan Pakiyaraja

University of Birmingham

Ben Duncombe

Badger Group

Daniel Lasserson

University of Warwick

\section{Research Article}

Keywords: COVID-19, primary care, clinical management

Posted Date: February 19th, 2021

DOl: https://doi.org/10.21203/rs.3.rs-211154/v1

License: (c) (i) This work is licensed under a Creative Commons Attribution 4.0 International License.

Read Full License 


\section{Abstract \\ Background}

The COVID-19 pandemic presented new diagnostic and management challenges to primary care alongside rapid changes in service delivery. The purpose of this study was to describe the characteristics of patients with symptoms of COVID-19 in primary care, explore which characteristics were associated with a clinical diagnosis of COVID-19 and referrals to secondary care, and to describe secondary care referral decisions with reference to national guidance.

\section{Methods}

An observational study using routinely collected data from the Birmingham Out-of-hours Research Database. The study uses consultation data from the Birmingham and Solihull COVID Referral Centre (CRC) between 21st April and 24th July. All CRC consultations were examined to extract patient demographics, free text consultations, prescriptions, observation and onward referrals. The National Early Warning Score (NEWS2) was calculated and the clinical diagnosis of COVID-19 was established. The population was described and univariate logistic regression was used to identify characteristics associated with clinical diagnosis of COVID-19 and referral decisions.

\section{Results}

681 patients were seen at the CRC and $56.3 \%$ were identified to have a clinical diagnosis of COVID-19. $14.0 \%$ of all patients were referred to secondary care, but $59 \%$ of patients classified as most severe according to national criteria were not referred. Referral was associated with increasing age, shortness of breath, tachycardia, tachypnoea and hypoxia but patients with a clinical COVID-19 diagnosis were less likely to be referred than those with other diagnoses.

\section{Conclusion}

Just over half of patients seen in the CRC were clinically diagnosed with COVID-19 and most patients were managed in the community. Guidelines developed in the absence of service delivery data for the management of COVID-19 were inconsistent with community urgent care delivery.

\section{Introduction}

In January 2020, the World Health Organisation recognised a novel coronavirus, Severe Acute Respiratory Syndrome Coronavirus 2 (SARS Co-V 2) and its associated disease, COVID-19, as a "Public Health Emergency of International Concern".(1) In the UK, the initial diagnosis of COVID-19 in primary care is made clinically as diagnostic testing is not available in this setting. The majority of patients have non- 
specific symptoms, such as cough, sore throat, fever, myalgia, arthralgia, fatigue, and headache, or are asymptomatic.(2) Therefore identifying the minority of patients with COVID-19 who may require hospitalisation is a new challenge to primary care clinicians.

UK general practice adapted quickly in response to the pandemic. This included undertaking most consultations remotely using telephone or video calls. $(3,4)$ New arrangements were also made for assessing potentially infectious patients in-person, and dedicated COVID-19 primary care hubs were established.(5) Birmingham and Solihull Clinical Commissioning Group, which commissions care for around 1.2M people in the West Midlands, UK,(6) established a COVID Referral Centre (CRC) to fulfil this role. General practitioners (GPs) remotely assessing patients with suspected COVID-19 had the options of managing patients at home, referring them to the CRC for further assessment, or referring them to secondary care acute services.

In June 2020, NHS England, the executive body that oversees the English NHS in the UK, published guidance for the assessment of patients with suspected COVID-19 in the community.(7) This classified the COVID-19 severity using physiological observations and indicated where patients should be managed. However, the distribution of COVID-19 severity in the community and the proportion of patients referred to hospital are currently unknown. Understanding how this guidance corresponds to real world practice would aid future planning of primary care service for pandemic responsiveness.

We aimed to describe the characteristics of patients who presented with symptoms of COVID-19 to primary care, explore which characteristics were associated with a clinical diagnosis of COVID-19 and referrals to secondary care, and to describe secondary care referral decisions with reference to national guidance.

\section{Methods}

\section{Study design}

This was an observational study using routine data from patient records within the Birmingham Out-ofhours general practice Research Database (BORD).

\section{Setting}

BORD contains all consultations undertaken by a large out-of-hours general practice provider (Badger Group) from July 2013 until July 2020 (inclusive). The Badger Group was commissioned to run the COVID Referral Centre (CRC) for patients in Birmingham and Solihull in the West Midlands, UK. Clinical encounters were recorded on the Adastra $\odot$ patient management system(8), which has no direct linkage to core hours general practice or secondary care systems.

\section{Participants}


We included all adults aged 16 years and older referred to the CRC who had a documented consultation. Patients were referred to the CRC using an electronic referral form [Additional file 1], which we used to identify eligible patients. Patients were referred if they needed further assessment in primary care and had symptoms of potential COVID-19. All patients meeting the study eligibility criteria were included.

\section{Variables}

The following variables were extracted: 1 ) demographic characteristics (age, sex, ethnic group and socioeconomic status [Townsend deprivation quintile(9)]); 2) symptoms on the referral from (cough, shortness of breath, fever and confusion); 3) risk factors on the referral from (resuscitation status, cardiovascular disease, diabetes mellitus, obesity, chronic obstructive pulmonary disease and classified as 'high risk' by the referring clinician); 4) physiological observations (temperature, respiratory rate, systolic blood pressure, oxygen saturation, and pulse rate); 5) prescriptions for oral antibiotics and corticosteroids; and 6 ) the postcode of the referring practice.

Physiological observations that were at the biological extremes were identified and their clinical plausibility checked through comparison with the rest of the record. If alternative observations were available in the free text, these were used to replace implausible values. Where no alternative was found, implausible values were regarded as data entry errors and were classed as missing. Patients with oxygen saturations below $96 \%$ were scrutinised to identify evidence of a diagnosis of chronic lung disease with baseline hypoxaemia. These patients were flagged and their recorded oxygen saturation was compared with their baseline or $88 \%$ if this was unavailable. Any evidence of prior use of long-term oxygen therapy was noted.

The National Early Warning Score 2 (NEWS2), a standardised aggregate measure of physiological disruption, was calculated for patients with the necessary observations.(10) Physiological observations and NEWS2 were used to stratify disease severity into mild, moderate or severe according to NHS England guidance [Additional file 2].(7) Although this pathway was designed for suspected COVID-19 patients only, the criteria was applied to all included patients for comparative purposes.

\section{Outcomes}

The outcomes of interest were the clinical diagnosis of COVID-19 and referral to secondary care. Records were evaluated to establish whether the consulting clinician had made a diagnosis of possible or probable COVID-19. A probable diagnosis of COVID-19 was coded where records documented that COVID19 was either the main diagnosis or among the top differential diagnoses. A possible diagnosis of COVID-19 was coded where records documented that the patient had been advised to self-isolate and/or seek testing for COVID-19 even if COVID-19 was not explicitly diagnosed. Outcomes independently coded by two clinical authors, with disagreements resolved by consensus between four clinical authors. Referrals to secondary care were identified from the recorded consultation outcome using methods previously described.(11) 


\section{Statistical analysis}

The number of referrals from individual practices were plotted geospatially using Microsoft Excel and Google Maps. The demographic and clinical characteristics of the study population were described using summary statistics, stratified by clinical COVID-19 diagnosis. The differences between these groups were tested using Chi squared tests, with the threshold for statistical significance adjusted using the Bonferroni correction.

A mixed effects logistic regression model was used to assess the univariate association between patient characteristics and clinical diagnosis of COVID-19, with the assessing clinician added as a random effect. Variables with missing data included a missing category and a complete case analysis was performed. The same methods were used to identify factors associated with secondary care referral decisions. Statistical analyses were performed using STATA14@.

\section{Results}

\section{Referrals and assessments}

There were $700 \mathrm{CRC}$ referrals between 21st April and 24th July (inclusive). Eleven were for children and eight had no clinical data and were assumed to have not been assessed by a clinician, leaving 681 clinical records in our study population.

These referrals originated from 131 different primary care practices with a median number of referrals of 2 (range 1-52, IQR 1-6). Referrals were generally spread throughout the catchment area without any clear geographic patterns (Fig. 1).

The frequency of referrals over time is shown in Fig. 2. More than two thirds of patients were assessed prior to the publication of NHS England COVID-19 assessment guidance(7) on 11th June. The consultations were undertaken by 79 clinicians with the median number of consultations per clinician being 41 (IQR 16 to 59 ) with $28 \%$ of clinicians having undertaken $71 \%$ of assessments.

\section{Participants}

Sociodemographic characteristics were similar between patients who were diagnosed with COVID-19 and alternative conditions (Table 1). Of the 7 patients reattending within 7 days, 5 were diagnosed with COVID-19 on initial presentation, and one more received this diagnosis on reattendance.

A higher proportion of patients diagnosed with suspected or probable COVID-19 than those who received alternative diagnoses had tachycardia ( $37.3 \%$ vs $33.6 \%$, respectively), fever ( $12.5 \%$ vs $6.4 \%$ ), tachypnoea ( $27.2 \%$ vs $20.5 \%$ ) and hypotension ( $20.4 \%$ vs $17.8 \%$ ), although these differences did not meet the adjusted threshold for statistical significance. Oxygen saturations below $96 \%$ was more common in patients who received an alternative diagnosis (13.8\% vs $11.8 \%$, respectively). The distribution of NEWS2 was similar between both groups 
Table 1

Participant characteristics, stratified by clinician diagnosis of COVID-19

\begin{tabular}{|c|c|c|c|c|c|c|c|c|}
\hline & & \multicolumn{4}{|c|}{$\begin{array}{l}\text { COVID-19 suspected or } \\
\text { diagnosed }\end{array}$} & \multirow{2}{*}{\multicolumn{2}{|c|}{$\begin{array}{l}\text { Total } \\
(n=681)\end{array}$}} & \multirow[b]{3}{*}{$P * \star$} \\
\hline & & \multicolumn{2}{|c|}{ Yes $(n=383)$} & \multicolumn{2}{|c|}{ No $(n=298)$} & & & \\
\hline & & $\mathbf{N}$ & $(\%)$ & $\mathbf{N}$ & $(\%)$ & $\mathbf{N}$ & $(\%)$ & \\
\hline Age group (years) & $16-29$ & 59 & $(15.4)$ & 43 & $(14.4)$ & 102 & $(15.0)$ & 0.117 \\
\hline & $30-44$ & 91 & $(23.8)$ & 68 & $(22.8)$ & 159 & $(23.4)$ & \\
\hline & $45-59$ & 125 & $(32.6)$ & 81 & $(27.2)$ & 206 & $(30.3)$ & \\
\hline & $60-74$ & 84 & $(21.9)$ & 72 & $(24.2)$ & 156 & $(22.9)$ & \\
\hline & $75+$ & 24 & $(6.3)$ & 34 & $(11.4)$ & 58 & (8.5) & \\
\hline Sex & Female & 254 & $(66.3)$ & 187 & $(62.8)$ & 441 & $(64.8)$ & 0.369 \\
\hline & Male & 128 & $(33.4)$ & 109 & $(36.6)$ & 237 & $(34.8)$ & \\
\hline & Missing & 1 & $(0.3)$ & 2 & $(0.7)$ & 3 & $(0.4)$ & \\
\hline Townsend quintile (1st & $1 \mathrm{st}$ & 34 & $(8.9)$ & 24 & $(8.1)$ & 58 & $(8.5)$ & 0.911 \\
\hline deprivation) & $2 n d$ & 42 & $(11.0)$ & 34 & $(11.4)$ & 76 & $(11.2)$ & \\
\hline & $3 r d$ & 65 & $(17.0)$ & 56 & $(18.8)$ & 121 & $(17.8)$ & \\
\hline & 4th & 90 & $(23.5)$ & 62 & $(20.8)$ & 152 & $(22.3)$ & \\
\hline & 5 th & 136 & $(35.5)$ & 112 & $(37.6)$ & 248 & $(36.4)$ & \\
\hline & Missing & 16 & $(4.2)$ & 10 & (3.4) & 26 & (3.8) & \\
\hline Referral symptoms & Fever & 170 & $(44.4)$ & 118 & $(39.6)$ & 288 & $(42.3)$ & 0.210 \\
\hline & Cough & 272 & $(71.0)$ & 179 & $(60.1)$ & 451 & $(66.2)$ & 0.003 \\
\hline & Dyspnoea & 250 & $(65.3)$ & 165 & $(55.4)$ & 415 & $(60.9)$ & 0.009 \\
\hline & Confusion & 5 & (1.3) & 1 & $(0.3)$ & 6 & $(0.9)$ & 0.179 \\
\hline Risk factors & High risk* & 25 & $(6.5)$ & 16 & (5.4) & 41 & $(6.0)$ & 0.528 \\
\hline & $\begin{array}{l}\text { Cardiovascular } \\
\text { disease }\end{array}$ & 25 & $(6.5)$ & 25 & (8.4) & 50 & (7.3) & 0.355 \\
\hline & Diabetes & 27 & $(7.1)$ & 24 & $(8.1)$ & 51 & $(7.5)$ & 0.621 \\
\hline $\begin{array}{l}\mathrm{BMI}=\text { body mass index, } \\
\text { Warning Score } 2, \mathrm{SES}=\end{array}$ & $\begin{array}{l}\mathrm{D}=\text { chronic ob } \\
\text { oeconomic sta }\end{array}$ & c & $\begin{array}{l}\text { Imon } \\
\text { Systol }\end{array}$ & $\begin{array}{l}\text { dise } \\
\text { Blooc }\end{array}$ & $\begin{array}{l}\text { e, NEWs } \\
\text { essure }\end{array}$ & $=1$ & nal & \\
\hline
\end{tabular}




\begin{tabular}{|c|c|c|c|c|c|c|c|c|}
\hline & \multirow[b]{2}{*}{ COPD } & \multicolumn{4}{|c|}{$\begin{array}{l}\text { COVID-19 suspected or } \\
\text { diagnosed }\end{array}$} & \multicolumn{3}{|c|}{ Total } \\
\hline & & 72 & $(18.8)$ & 44 & $(14.8)$ & 116 & $(17.0)$ & 0.165 \\
\hline & $\mathrm{BMI}>30$ & 44 & $(11.5)$ & 32 & $(10.7)$ & 76 & $(11.2)$ & 0.758 \\
\hline \multirow[t]{5}{*}{ Observations } & $\begin{array}{l}\text { Pulse rate > } 90 \\
\text { bpm }\end{array}$ & 143 & (37.3) & 100 & $(33.6)$ & 243 & (35.7) & 0.307 \\
\hline & $\begin{array}{l}\text { Temperature }> \\
37.8\end{array}$ & 48 & $(12.5)$ & 19 & $(6.4)$ & 67 & (9.8) & 0.007 \\
\hline & $\begin{array}{l}\text { Respiratory } \\
\text { rate }>20\end{array}$ & 104 & $(27.2)$ & 61 & $(20.5)$ & 165 & $(24.2)$ & 0.043 \\
\hline & $\begin{array}{l}\text { Oxygen } \\
\text { saturation < } \\
96 \%\end{array}$ & 45 & $(11.8)$ & 41 & $(13.8)$ & 86 & $(12.6)$ & 0.434 \\
\hline & $\begin{array}{l}\mathrm{SBP}<110 \\
\mathrm{mmHg}\end{array}$ & 78 & $(20.4)$ & 53 & $(17.8)$ & 131 & (19.2) & 0.397 \\
\hline \multirow[t]{7}{*}{ NEWS2 } & 0 & 147 & $(38.4)$ & 131 & $(44.0)$ & 278 & $(40.8)$ & 0.799 \\
\hline & 1 & 101 & $(26.4)$ & 70 & $(23.5)$ & 171 & $(25.1)$ & \\
\hline & 2 & 53 & $(13.8)$ & 39 & $(13.1)$ & 92 & $(13.5)$ & \\
\hline & 3 & 26 & $(6.8)$ & 20 & $(6.7)$ & 46 & (6.8) & \\
\hline & 4 & 14 & (3.7) & 9 & $(3.0)$ & 23 & (3.4) & \\
\hline & $5+$ & 15 & (3.9) & 6 & $(2.0)$ & 21 & (3.1) & \\
\hline & Missing & 27 & $(7.1)$ & 23 & (7.7) & 50 & (7.3) & \\
\hline \multirow[t]{3}{*}{ Severity *** } & Mild & 214 & $(55.9)$ & 187 & $(62.8)$ & 401 & $(58.9)$ & 0.169 \\
\hline & Moderate & 147 & $(38.4)$ & 94 & $(31.5)$ & 241 & $(35.4)$ & \\
\hline & Severe & 22 & (5.7) & 17 & $(5.7)$ & 39 & (5.7) & \\
\hline \multirow[t]{2}{*}{ Prescriptions } & Antibiotics & 141 & $(36.8)$ & 70 & $(23.5)$ & 211 & $(31.0)$ & ¿. 001 \\
\hline & Corticosteroids & 50 & $(13.1)$ & 15 & $(5.0)$ & 65 & (9.5) & $\hat{0}_{0.001}$ \\
\hline Referred & & 42 & $(11.0)$ & 53 & (17.8) & 95 & $(14.0)$ & 0.011 \\
\hline
\end{tabular}

$\mathrm{BMI}=$ body mass index, $\mathrm{COPD}=$ chronic obstructive pulmonary disease, NEWS2 $=$ National Early Warning Score 2, SES = socioeconomic status, SBP = Systolic Blood Pressure

*Deemed by referring clinician to be at high clinical risk, **Threshold for statistical significance corrected to 0.002 using the Bonferroni correction, *** Severity graded according to NHSE algorithm 


\begin{tabular}{|c|c|c|c|c|c|c|c|}
\hline \multirow[b]{2}{*}{ Re-attendance } & \multicolumn{4}{|c|}{$\begin{array}{l}\text { COVID-19 suspected or } \\
\text { diagnosed }\end{array}$} & \multicolumn{3}{|c|}{ Total } \\
\hline & 6 & $(1.57)$ & 1 & $(0.34)$ & 7 & $(1.03)$ & 0.114 \\
\hline \multicolumn{8}{|c|}{$\begin{array}{l}\mathrm{BMI}=\text { body mass index }, \mathrm{COPD}=\text { chronic obstructive pulmonary disease, NEWS2 = National Early } \\
\text { Warning Score } 2, \mathrm{SES}=\text { socioeconomic status, SBP = Systolic Blood Pressure }\end{array}$} \\
\hline
\end{tabular}

\section{Outcomes}

383 of 681 patients (56.3\%) had a clinical diagnosis of possible or probable COVID-19 (125 (18.4\% possible, $258(37.9 \%)$ probable). These patients were significantly more likely to receive antibiotics and oral corticosteroids than those who received alternative diagnoses $(36.8 \%$ vs $23.5 \%, p<0.001$ for antibiotics and $13.1 \%$ vs $5.0 \%, p<0.001$ for steroids; Table 1 ).

In total, $14.0 \%$ of patients were referred to secondary care; $11.0 \%$ of those with possible or probable COVID-19 and $17.8 \%$ of those with other diagnoses. The characteristics of referred patients are shown in [Additional file 3]. The referral rate to secondary care increased with severity of illness but was still only $41.0 \%$ of those in the most severe category (Fig. 3 ).

\section{Main results}

The factors significantly associated with receiving a clinical diagnosis of possible or probable COVID-19 were presence of cough (OR $1.74,95 \% \mathrm{Cl} 1.22$ to 2.48$)$, dyspnoea $(1.64,1.17$ to 2.31$)$, and pyrexia (2.14, 1.17 to $3.90 ;$ Table 2). Similar findings were found in the sensitivity analysis where the outcome was restricted to cases of probable COVID-19 [Additional file 4]. 
Table 2

Unadjusted odds ratios (OR) for diagnosis of suspected or probable COVID-19

\begin{tabular}{|c|c|c|c|c|}
\hline Risk factors & & OR & $(95 \% \mathrm{Cl})$ & $p$ \\
\hline \multirow[t]{5}{*}{ Age (years) } & $16-29$ & \multicolumn{3}{|c|}{ (reference) } \\
\hline & $30-44$ & 0.95 & $\begin{array}{l}(0.55 \\
1.64)\end{array}$ & 0.860 \\
\hline & $45-59$ & 1.16 & $\begin{array}{l}(0.69 \\
1.96)\end{array}$ & 0.570 \\
\hline & $60-74$ & 0.90 & $\begin{array}{l}(0.52 \\
1.56)\end{array}$ & 0.704 \\
\hline & $75+$ & 0.46 & $\begin{array}{l}(0.22 \\
0.95)\end{array}$ & 0.036 \\
\hline \multirow[t]{2}{*}{ Sex } & Male & \multicolumn{3}{|c|}{ (reference) } \\
\hline & Female & 1.09 & $\begin{array}{l}(0.77 \\
1.54)^{\prime}\end{array}$ & 0.622 \\
\hline \multirow{6}{*}{$\begin{array}{l}\text { Townsend quintile (1st representing lowest } \\
\text { deprivation) }\end{array}$} & $1 \mathrm{st}$ & \multicolumn{3}{|c|}{ (reference) } \\
\hline & 2nd & 1.01 & $\begin{array}{l}(0.47 \\
2.15)\end{array}$ & 0.979 \\
\hline & $3 r d$ & 0.85 & $\begin{array}{l}(0.42 \\
1.70)\end{array}$ & 0.645 \\
\hline & 4 th & 1.09 & $\begin{array}{l}(0.56 \\
2.14)\end{array}$ & 0.800 \\
\hline & 5th & 0.87 & $\begin{array}{l}(0.46 \\
1.64)\end{array}$ & 0.671 \\
\hline & Missing & 1.07 & $\begin{array}{l}(0.38 \\
3.00)\end{array}$ & 0.902 \\
\hline \multirow[t]{4}{*}{ Symptoms } & Fever & 1.19 & $\begin{array}{l}(0.85 \\
1.66)\end{array}$ & 0.320 \\
\hline & Cough & 1.74 & $\begin{array}{l}(1.22, \\
2.48)\end{array}$ & 0.002 \\
\hline & Dyspnoea & 1.64 & $\begin{array}{l}(1.17 \\
2.31)\end{array}$ & 0.005 \\
\hline & Confusion & 4.68 & $\begin{array}{l}(0.48 \\
46.98)\end{array}$ & 0.186 \\
\hline Risk factors & High risk & 1.13 & $\begin{array}{l}(0.56, \\
2.30)\end{array}$ & 0.727 \\
\hline
\end{tabular}

* Severity graded according to NHSE algorithm 


\begin{tabular}{|c|c|c|c|c|}
\hline \multicolumn{2}{|l|}{ Risk factors } & \multirow{2}{*}{$\begin{array}{l}\text { OR } \\
0.73\end{array}$} & \multirow{2}{*}{$\begin{array}{l}(95 \% \mathrm{Cl}) \\
(0.38 \\
1.40)\end{array}$} & \multirow{2}{*}{$\begin{array}{l}\mathbf{p} \\
0.347\end{array}$} \\
\hline & CVD & & & \\
\hline & Diabetes & 0.77 & $\begin{array}{l}(0.41 \\
1.44)\end{array}$ & 0.418 \\
\hline & COPD & 1.27 & $\begin{array}{l}(0.81 \\
2.00)\end{array}$ & 0.294 \\
\hline & $\mathrm{BMI}>30$ & 1.02 & $\begin{array}{l}(0.60 \\
1.73)\end{array}$ & 0.937 \\
\hline \multirow[t]{5}{*}{ Observations } & Pulse $>90 \mathrm{bpm}$ & 1.07 & $\begin{array}{l}(0.75 \\
1.51)\end{array}$ & 0.722 \\
\hline & $\mathrm{T}>37.8^{\circ} \mathrm{C}$ & 2.14 & $\begin{array}{l}(1.17 \\
3.90)\end{array}$ & 0.013 \\
\hline & $\mathrm{RR}>20$ & 1.46 & $\begin{array}{l}(0.98 \\
2.16)\end{array}$ & 0.064 \\
\hline & $\mathrm{Sa} 02<96 \%$ & 0.82 & $\begin{array}{l}(0.50 \\
1.35)\end{array}$ & 0.443 \\
\hline & $\begin{array}{l}\mathrm{SBP}<110 \\
\mathrm{mmHg}\end{array}$ & 1.08 & $\begin{array}{l}(0.70 \\
1.65)\end{array}$ & 0.737 \\
\hline \multirow[t]{6}{*}{ NEWS2 } & 0 & \multicolumn{3}{|c|}{ (reference) } \\
\hline & 1 & 1.18 & $\begin{array}{l}(0.77 \\
1.81)^{\prime}\end{array}$ & 0.448 \\
\hline & 2 & 1.08 & $\begin{array}{l}(0.64, \\
1.83)\end{array}$ & 0.771 \\
\hline & 3 & 1.12 & $\begin{array}{l}(0.56 \\
2.23)\end{array}$ & 0.757 \\
\hline & 4 & 1.24 & $\begin{array}{l}(0.48 \\
3.22)\end{array}$ & 0.659 \\
\hline & $5+$ & 2.08 & $\begin{array}{l}(0.69 \\
6.26)\end{array}$ & 0.193 \\
\hline \multirow[t]{3}{*}{ Severity* } & Mild & \multicolumn{3}{|c|}{ (reference) } \\
\hline & Moderate & 1.24 & $\begin{array}{l}(0.87 \\
1.76)\end{array}$ & 0.243 \\
\hline & Severe & 0.99 & $\begin{array}{l}(0.48 \\
2.07)\end{array}$ & 0.986 \\
\hline * Severity grad & & & & \\
\hline
\end{tabular}

Mixed effects logistic regression used to account for clustering by clinician 
$\mathrm{BPM}=$ beats per minute, $\mathrm{Cl}=$ confidence interval, $\mathrm{Sa02}=$ oxygen saturations, $\mathrm{SBP}=$ systolic blood pressure

Referral to secondary care was significantly associated with older age (OR 6.38,95\% Cl 1.96 to 20.8 in those aged $\geq 75$ years compared to those aged 16-29), shortness of breath (1.88, 1.06 to 3.35 ), history of diabetes (3.26, 1.24 to 8.55$)$, tachycardia $(2.25,1.27$ to 3.98$)$, tachypnoea $(5.15,2.72$ to 9.73$)$, and hypoxia (3.48, 1.71 to 7.06 ; Table 3$)$. A diagnosis of possible or probable COVID-19 was associated with a reduced odds of referral to secondary care compared to an alternative diagnosis (OR $0.54,95 \% \mathrm{Cl} 0.30$ to 0.97). 
Table 3

Unadjusted odds ratios (OR) for referral to secondary care

\begin{tabular}{|c|c|c|c|c|}
\hline \multicolumn{2}{|l|}{ Risk factors } & \multirow{2}{*}{\multicolumn{2}{|c|}{$\begin{array}{l}\text { OR } \quad(95 \% \mathrm{Cl}) \\
\text { (reference) }\end{array}$}} & \multirow[t]{2}{*}{ p } \\
\hline \multirow[t]{5}{*}{ Age (years) } & $16-29$ & & & \\
\hline & $30-44$ & 0.90 & $(0.31,2.56)$ & 0.837 \\
\hline & $45-59$ & 2.64 & $(1.01,6.88)$ & 0.048 \\
\hline & $60-74$ & 2.54 & $(0.96,6.77)$ & 0.062 \\
\hline & $75+$ & 6.38 & $(1.96,20.8)$ & 0.002 \\
\hline \multirow[t]{2}{*}{ Sex } & Male & \multicolumn{3}{|c|}{ (reference) } \\
\hline & Female & 1.22 & $(0.69,2.18)$ & 0.493 \\
\hline \multirow{6}{*}{$\begin{array}{l}\text { Townsend quintile ( } 1 \text { st representing lowest } \\
\text { deprivation) }\end{array}$} & $1 \mathrm{st}$ & \multicolumn{3}{|c|}{ (reference) } \\
\hline & $2 n d$ & 0.83 & $(0.24,2.79)$ & 0.758 \\
\hline & $3 r d$ & 0.83 & $(0.26,2.61)$ & 0.750 \\
\hline & 4 th & 1.15 & $(0.40,3.33)$ & 0.794 \\
\hline & 5th & 1.43 & $(0.54,3.79)$ & 0.471 \\
\hline & Missing & 1.75 & $(0.31,9.82)$ & 0.525 \\
\hline \multirow[t]{4}{*}{ Symptoms } & Fever & 1.47 & $(0.84,2.56)$ & 0.175 \\
\hline & Cough & 0.60 & $(0.34,1.05)$ & 0.076 \\
\hline & SOB & 1.88 & $(1.06,3.35)$ & 0.032 \\
\hline & Confusion & 3.36 & $(0.36,31.2)$ & 0.287 \\
\hline \multirow[t]{5}{*}{ Risk factors } & High risk & 2.02 & $(0.75,5.41)$ & 0.164 \\
\hline & CVD & 1.44 & $(0.53,3.92)$ & 0.477 \\
\hline & Diabetes & 3.26 & $(1.24,8.55)$ & 0.016 \\
\hline & COPD & 1.31 & $(0.64,2.71)$ & 0.463 \\
\hline & $\mathrm{BMI}>30$ & 0.79 & $(0.33,1.89)$ & 0.599 \\
\hline \multirow[t]{3}{*}{ Observations } & Pulse $>90$ & 2.25 & $(1.27,3.98)$ & 0.006 \\
\hline & $\mathrm{T}>37.8$ & 4.88 & $(2.24,10.7)$ & $<.001$ \\
\hline & $\mathrm{RR}>20$ & 5.15 & $(2.72,9.73)$ & $\dot{0}_{0.001}$ \\
\hline
\end{tabular}




\begin{tabular}{|c|c|c|c|c|}
\hline \multicolumn{2}{|l|}{ Risk factors } & \multirow{2}{*}{$\begin{array}{l}\text { OR } \\
3.48\end{array}$} & \multirow{2}{*}{$\begin{array}{l}(95 \% \mathrm{Cl}) \\
(1.71,7.06)\end{array}$} & \multirow{2}{*}{$\begin{array}{l}\mathbf{p} \\
0.001\end{array}$} \\
\hline & $\mathrm{Sa02}<96 \%$ & & & \\
\hline & $\mathrm{SBP}<110$ & 1.77 & $(0.89,3.54)$ & 0.106 \\
\hline \multirow[t]{6}{*}{ NEWS2 } & 0 & \multicolumn{3}{|c|}{ (reference) } \\
\hline & 1 & 1.27 & $(0.53,3.00)$ & 0.591 \\
\hline & 2 & 4.65 & $(1.86,11.6)$ & 0.001 \\
\hline & 3 & 7.74 & $(2.51,23.9)$ & $\begin{array}{l}< \\
0.001\end{array}$ \\
\hline & 4 & 15.05 & $(2.90,78.2)$ & 0.001 \\
\hline & $5+$ & 40.69 & $\begin{array}{l}(8.98, \\
184.5)\end{array}$ & $\begin{array}{l}<.001 \\
0.001\end{array}$ \\
\hline \multirow[t]{3}{*}{ Severity* } & Mild & \multicolumn{3}{|c|}{ (reference) } \\
\hline & Moderate & 3.60 & $(1.90,6.81)$ & $\begin{array}{l}< \\
0.001\end{array}$ \\
\hline & Severe & 25.50 & $(7.96,81.7)$ & $\dot{0}_{0.001}$ \\
\hline \multirow[t]{2}{*}{ Clinical Diagnosis } & $\begin{array}{l}\text { Non-Covid- } \\
19\end{array}$ & \multicolumn{3}{|c|}{ (reference) } \\
\hline & Covid-19 & 0.54 & $(0.30,0.97)$ & 0.039 \\
\hline
\end{tabular}

Mixed effects logistic regression used to account for clustering by clinician

$\mathrm{BPM}=$ beats per minute $\mathrm{Cl}=$ confidence interval, $\mathrm{Sa02}=$ oxygen saturations, $\mathrm{SBP}=$ systolic blood pressure

\section{Discussion}

Only just over half of patients referred to the CRC due to COVID-19 symptoms were given a clinical diagnosis of possible or probable COVID-19. Presence of cough, dyspnoea and pyrexia were associated with a higher odds of being clinically diagnosed with COVID-19 in the absence of confirmatory testing. This is unsurprising given that these were highlighted as key symptoms of COVID-19 early on during the pandemic. By contrast, hypoxia was not associated with suspicion of COVID-19, which is inconsistent with the prominence of lower oxygen saturations in clinical guidelines. Patients with suspected COVID-19 were more likely to be prescribed antibiotics and steroids, neither or which are supported by clinical guidelines. This may in part be due to clinical uncertainty early on in the pandemic or may reflect a heuristic of wanting to intervene in these patients, especially since they were less likely to be referred to 
hospital. The relatively high prevalence of COPD in this patient population may also have contributed to this finding.

Only a minority of patients referred to the CRC were referred to secondary care and this was more likely among those who were objectively more severely ill (i.e. had a higher NEWS2). Older patients, those with tachypnoea, hypoxia, tachycardia, diabetes and shortness of breath had a significantly higher odds of being referred to secondary care following clinical assessment. However, patients with a clinical diagnosis of COVID-19 were less likely to be referred, which may have been due to a perceived lack of therapeutic options in secondary care.

$81.7 \%$ of patients with moderate severity illness (where referral to secondary care should be considered) were managed in the community along with $59 \%$ of patients in the highest severity category. There was, therefore, a discrepancy between the judgement of clinicians and the recommended referral thresholds from national protocols. This may be because the severity of illness was under-recognised by clinicians, or that the clinical risk assessment of severity differed from the thresholds recommended in the protocols. Alternatively, the option of hospital referral may have been offered to patients with moderateto-severe illness, but community management was agreed upon through shared decision making. At the time, here was considerable fear about hospitalisation, which may have influenced patient preferences on location of care; and clinicians may have preferred managing patients in the community due to concerns around overwhelming secondary care. Finally, patients managed in the community will have been given safety-netting advice including when to attend secondary care, however we were unable to identify patients who subsequently attended secondary care in the same period of illness.

Despite major restructuring of primary care during the pandemic, $(12,13)$ little is known about service usage and characteristics of patients with COVID-19 in the community. The Birmingham CRC was established to cope with a worst-case scenario of up to 500 patients/day,(14) but in practice the busiest week saw fewer than 90 patients assessed. However, the system was designed to allow a dynamic response to demand in order to reduce waste and optimise resources. There was no evidence from the geographical spread of referrals that certain areas of the region were under-represented even though patients did need to arrange their own transport to attend the CRC. The spectrum of socioeconomic deprivation among patients who attended the $\mathrm{CRC}$ also suggests that the service was used equitably across the Birmingham population.

\section{Comparisons with existing research}

There are no comparable studies investigating how patients with suspected COVID-19 are assessed and triaged in primary care setting.

We found that $66 \%$ of patients with suspected COVID-19 were female. Other studies in hospital and primary care settings found a male predominance.(15-19) This may reflect differences in health-seeking behaviour, where males are less likely to access primary care, as well as the spectrum of illness severity with men potentially presenting with more severe disease directly to secondary care. 
The International Severe Acute Respiratory and emerging Infections Consortium (ISARIC) described UK patients admitted to hospital.(15) Only $28.2 \%$ of patients with suspected COVID-19 at the CRC were 60 years or older compared with $72.7 \%$ of those in the ISARIC study. Older age has clearly emerged as one of the most important risk factors for severe COVID-19,(17, 19) which correlates with our findings.

The proportion of patients presenting with symptoms of cough and shortness of breath at the CRC were comparable to those presenting to secondary care(15) although patients with suspected COVID-19 assessed at the CRC were less likely to report fever (44\% at the CRC vs $72 \%)$.

When the same database was used to analyse pre-pandemic face-to-face out-of-hours consultations a similar sex and socioeconomic deprivation distribution was found, but the patients were younger than those seen at the CRC (mean age 40.6 years versus 49.6 respectively).(20) Referral rates were higher in the CRC ( $8.5 \%$ vs $14.0 \%$ respectively) and patients assessed at the CRC were generally more unwell with the proportion of patients with a NEWS 2 of $\geq 5$ being $3.1 \%$ at the CRC compared with $2.0 \%$ in standard out-of-hours care and the respective proportions of those with a NEWS2 of 0 or 1 was $77.5 \%$ and $65.9 \%$. This is consistent with the differential referral rates to secondary care.

\section{Strengths and limitations}

This is the first study to describe the characteristics of patients referred to a COVID-19 primary care referral centre and how guidance for escalation of care was applied in a rapidly adapting primary care system. It was not possible to link this data with diagnostic testing for COVID-19 so conclusions can only be drawn based on clinical suspicion of infection, which represents the reality of current UK general practice. Further outcomes for patients either referred into hospital or advised to self-manage at home are not available in this dataset so we were unable to comment on the clinical consequences of referral decisions. Finally, patients were triaged by a primary care clinician before referral to the CRC, and we did not have data on severely ill patients with COVID-19 who were identified at this stage and advised to attend secondary care or those advised to self-manage at home without being referred to the CRC.

\section{Implications for practice, policy and research}

Utilisation of the CRC was less than anticipated, which highlights the importance of a flexible primary care system capable of escalating and de-escalating its response in a dynamic pandemic situation. Our findings also highlight that primary care CRCs should be prepared to receive a high proportion of patients with non-COVID-19 diagnoses, even with prior clinical triage.

We also have identified discrepancies between clinical guidelines for COVID-19 and observed practice. However, whether guidelines should better reflect the abilities of clinicians to assess and manage risk, or whether clinicians should more stringently apply guidelines is difficult to ascertain from available data. Further iteration and validation of clinical guidelines should be considered.

Finally, there is still a need to better understand the natural history of COVID-19 in the community. Further studies investigating the physiology and outcomes of patients managed in the community would 
improve our ability to meet the needs of this large public health challenge.

\section{Conclusion}

Although the CRC was not utilised as much as anticipated, it provided an important role in assessing patients with suspected COVID-19 and the majority of these patients were not thought to have COVID-19 after assessment. Regardless of diagnosis, most patients were managed in the community, thereby reducing pressure on secondary care. Patients seen in the CRC were more unwell than usual primary care patients and abnormal physiological observations were associated with referral to hospital.

\section{List Of Abbreviations}

CRC

COVID Referral Centre

BORD

Birmingham Out-of-hours Research Database

GP

General Practitioner

NEWS2

National Early Warning Score version 2

$\mathrm{NHS}$

National Health Service (England)

\section{Declarations}

Ethical approval: Research using BORD was approved by the South Birmingham Research Ethics Committee (ref. 19/WM/0010)

Consent for publication: Not applicable

Availability of data and materials: The datasets generated and/or analysed during the current study are not publicly available due data sharing restrictions but are available from the corresponding author on reasonable request.

Competing interests: All authors have no competing interests to declare.

Funding: The study received no external funding. This study was supported by the National Institute of Health Research (NIHR) Applied Research Collaboration (ARC) West Midlands. JC is an NIHR funded Academic Clinical Fellow. AA is funded by Health Education England, West Midlands. The views expressed are of the authors and not necessarily those of the NIHR or Department of Health and Social Care. 
Authors' contributions: SF and SH conceived the idea for the study. SH and AP drafted the initial study protocol. BD extracted the data. SF cleaned the data. SF and SH performed the data analysis. JC and AA performed a literature review and drafted sections of the introduction and discussion. SF and SH drafted the main manuscript with input from JC, AA, and DL. AP produced figure 1. All authors reviewed and approved the final manuscript.

Acknowledgements: We would like to thank Dr Simon Dodds for reviewing our manuscript and providing helpful feedback. Thanks also to Solihull and Birmingham CCG for providing data on their member practices

\section{References}

1. World Health Organisation. Statement on the second meeting of the International Health Regulations (2005) Emergency Committee regarding the outbreak of novel coronavirus (2019-nCoV): World Health Organisation; 2020 [15.11.2020]. Available from: https://www.who.int/news/item/30-012020-statement-on-the-second-meeting-of-the-international-health-regulations-(2005)-emergencycommittee-regarding-the-outbreak-of-novel-coronavirus-(2019-ncov).

2. Struyf T, Deeks JJ, Dinnes J, Takwoingi Y, Davenport C, Leeflang MM, et al. Signs and symptoms to determine if a patient presenting in primary care or hospital outpatient settings has COVID-19 disease. Cochrane Database of Systematic Reviews. 2020(7).

3. Greenhalgh T, Koh GCH, Car J. Covid-19: a remote assessment in primary care. British Medical Journal. 2020;368:m1182.

4. Joy M, McGagh D, Jones $N$, Liyanage $H$, Sherlock J, Parimalanathan $\mathrm{V}$, et al. Reorganisation of primary care for older adults during COVID-19: a cross-sectional database study in the UK. British Journal of General Practice. 2020;70(697):e540-e7.

5. Majeed A, Maile EJ, Bindman AB. The primary care response to COVID-19 in England's National Health Service. Journal of the Royal Society of Medicine. 2020;113(6):208-10.

6. Birmingham and Solihull CCG. Birmingham and Solihull Clinical Commissioning Group: About us 2020 [15.11.2020]. Available from: https://www.birminghamandsolihullccg.nhs.uk/about-us.

7. NHS England. Pulse oximetry to detect early deterioration of patients with COVID-19 in primary and community care settings: NHS England; 2020 [10/08/2020]. Available from: https://www.england.nhs.uk/coronavirus/wp-content/uploads/sites/52/2020/06/C0445-remotemonitoring-in-primary-care.pdf.

8. Advanced. Adastra Clinical Patient Management Solutions [Available from: https://www.oneadvanced.com/products/adastra/.

9. Townsend P, Phillimore P, Beattie A. Health and deprivation: inequality and the North: Routledge; 1988.

10. Royal College of Physicians. National Early Warning Score (NEWS)2: Standardising the assessment of acute-illness severity in the NHS London: RCP; 2017 [06/12/2019]. Available from: 
https://www.rcplondon.ac.uk/projects/outputs/national-early-warning-score-news-2.

11. Finnikin S, Hayward G, Wilson F, Lasserson D. Are referrals to hospital from out-of-hours primary care associated with National Early Warning Scores? Emerg Med J. 2020.

12. Park S, Elliott J, Berlin A, Hamer-Hunt J, Haines A. Strengthening the UK primary care response to covid-19. bmj. 2020;370.

13. NHS England. Guidance and standard operating procedures: General practice in the context of coronavirus (COVID-19) 2020 [updated 10/08/202008/11/2020]. Available from: https://www.england.nhs.uk/coronavirus/wp-content/uploads/sites/52/2020/03/C0485_guidanceand-standard-operating-procedures-general-practice-covid-19.pdf.

14. Dodds S. BSOL CRC e-Referral Summary (Week 12) - Mon 13/07/2020. 2020.

15. Docherty AB, Harrison EM, Green CA, Hardwick HE, Pius R, Norman L, et al. Features of 20133 UK patients in hospital with covid-19 using the ISARIC WHO Clinical Characterisation Protocol: prospective observational cohort study. bmj. 2020;369.

16. Abate BB, Kassie AM, Kassaw MW, Aragie TG, Masresha SA. Sex difference in coronavirus disease (COVID-19): a systematic review and meta-analysis. BMJ open. 2020;10(10):e040129.

17. de Lusignan S, Dorward J, Correa A, Jones N, Akinyemi O, Amirthalingam G, et al. Risk factors for SARS-CoV-2 among patients in the Oxford Royal College of General Practitioners Research and Surveillance Centre primary care network: a cross-sectional study. The Lancet Infectious Diseases. 2020.

18. Ferreira-Santos $D$, Maranhão $P$, Monteiro-Soares $M$. Identifying common baseline clinical features of COVID-19: a scoping review. BMJ open. 2020;10(9):e041079.

19. Williamson EJ, Walker AJ, Bhaskaran K, Bacon S, Bates C, Morton CE, et al. Factors associated with COVID-19-related death using OpenSAFELY. Nature. 2020;584(7821):430-6.

20. Finnikin S, Hayward G, Wilson F, Lasserson D. Are referrals to hospital from out-of-hours primary care associated with National Early Warning Scores? Emergency Medicine Journal. 2020(37(5)):279-/85.

\section{Figures}




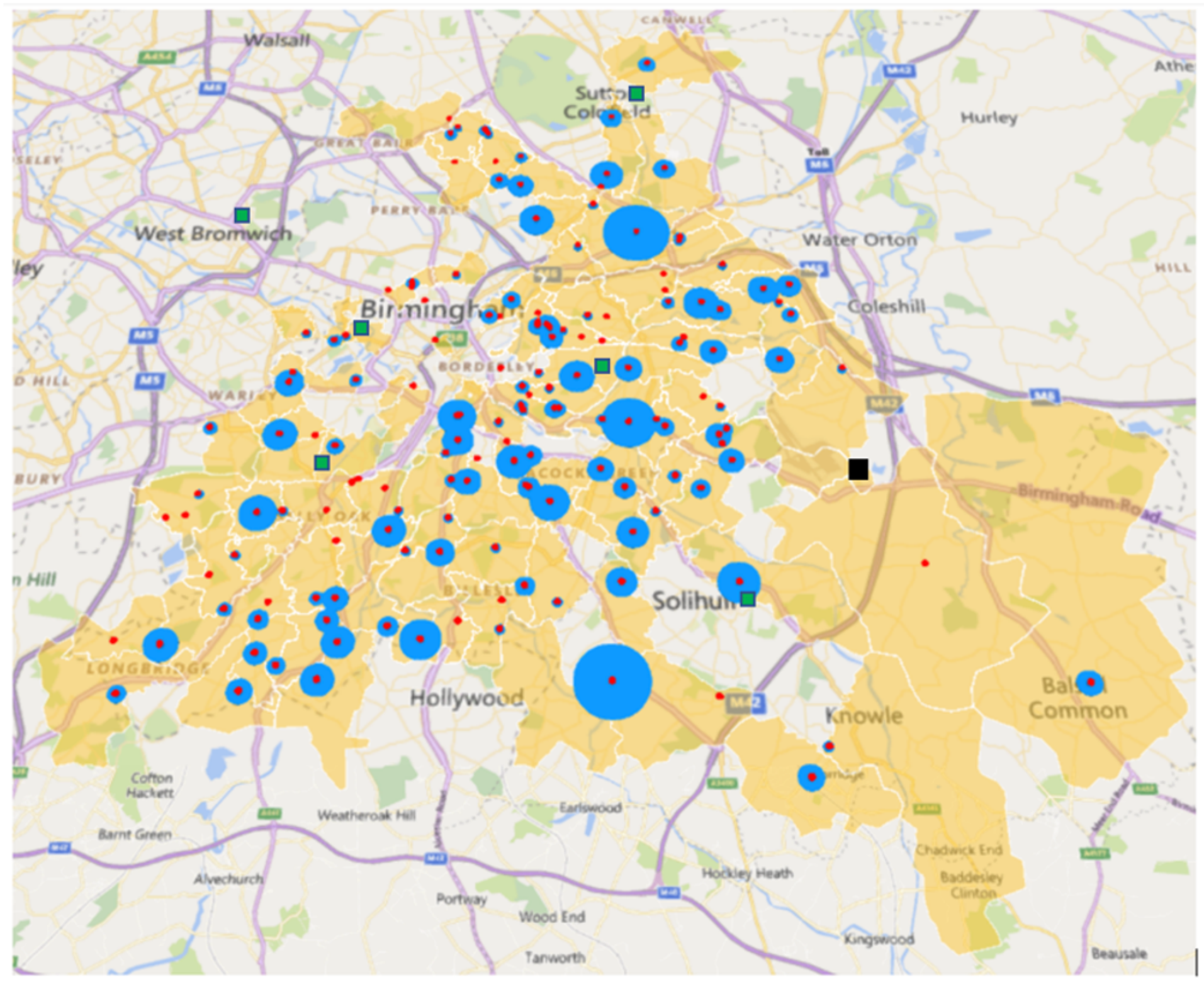

\section{Figure 1}

Locations of primary care practices in Birmingham and Solihull CCG. Practices are identified by red dots. The size of the blue circles represents the number of patients referred from that practice. The CRC is marked with a black square, and hospitals providing acute care are shown (green square) 


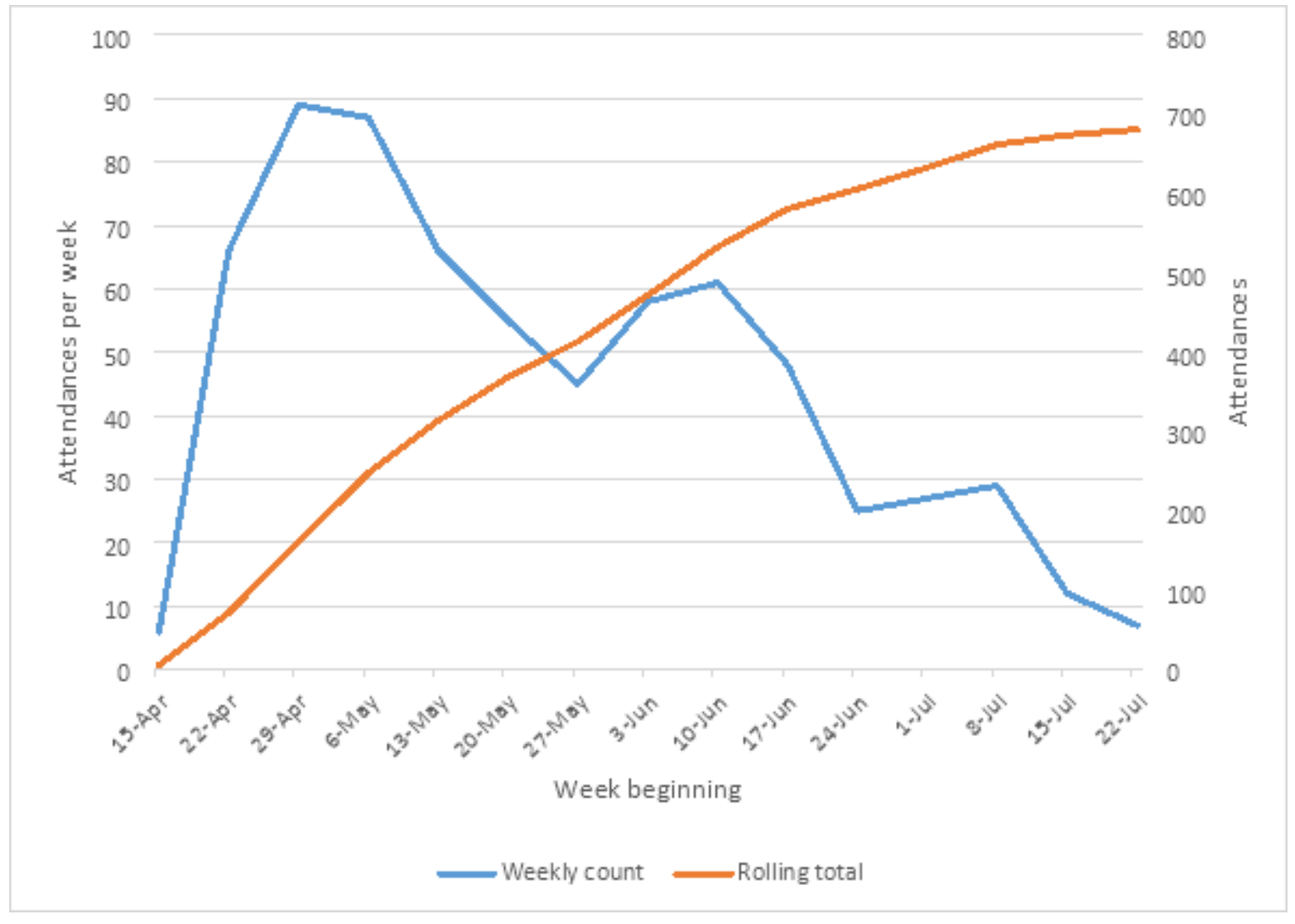

Figure 2

Frequency of attendances to the COVID-19 Referral Centre over time

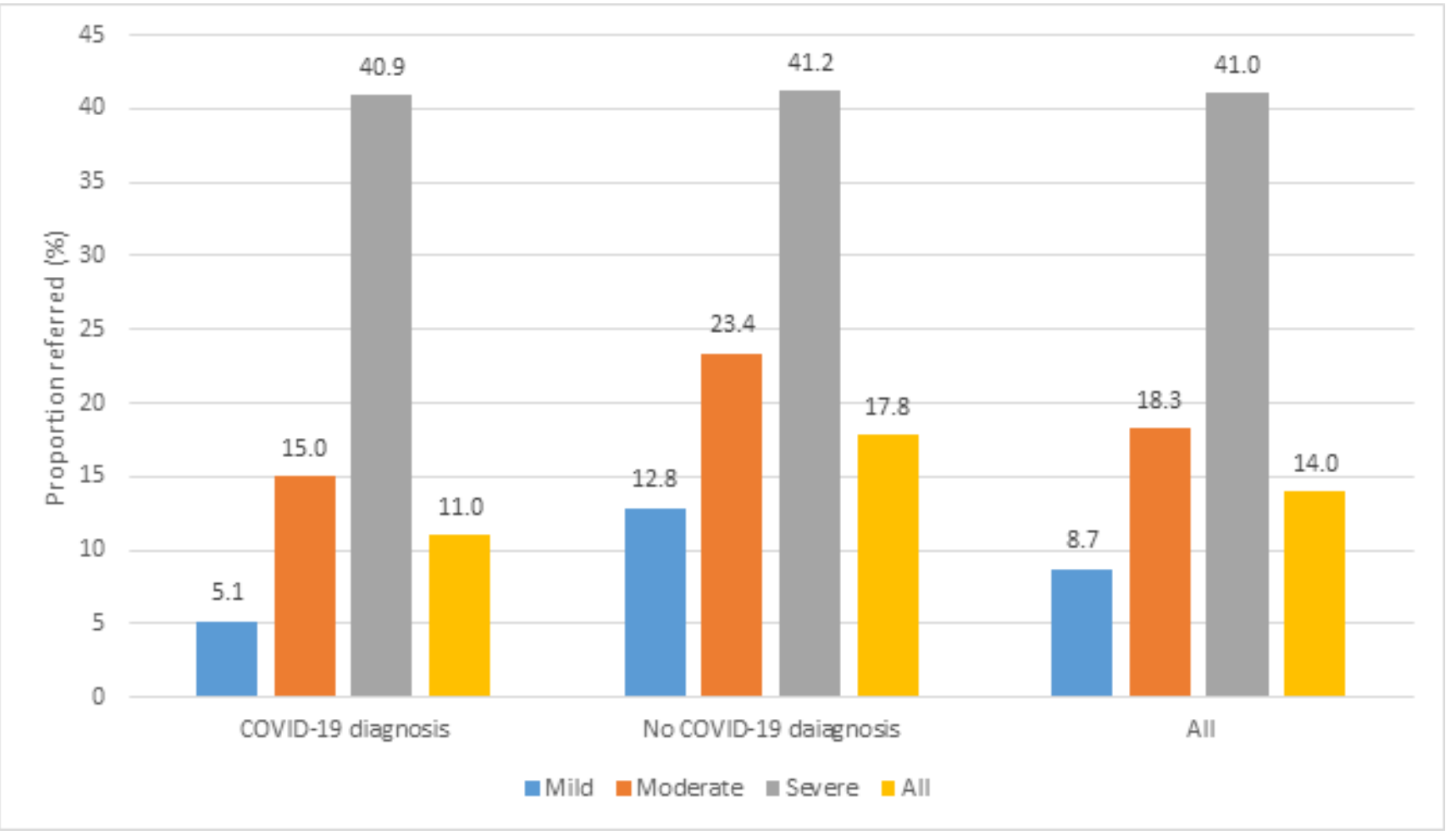

Figure 3 
Proportion of patients referred to secondary care from the CRC, by severity and diagnosis

\section{Supplementary Files}

This is a list of supplementary files associated with this preprint. Click to download.

- Additionalfile1.docx

- Additionalfile2.docx

- Additionalfile3.docx

- Additionalfile4.docx 